\title{
Natural immunity against capsular group X N. meningitidis following an outbreak in Togo, 2007
}

\author{
Gunnstein Norheim ${ }^{\mathrm{a}, \mathrm{b}, *}$, Judith E. Mueller ${ }^{\mathrm{c}, \mathrm{d}}$, Berthe-Marie Njanpop-Lafourcade ${ }^{\mathrm{c}}$, Isabelle Delrieu ${ }^{\mathrm{c}}$, \\ Helen Findlow $^{\mathrm{e}}$, Ray Borrow ${ }^{\mathrm{e}}$, Ouli Xie ${ }^{\mathrm{a}}$, Jerry Nagaputra ${ }^{\mathrm{a}}$, Roshan Ramasamy ${ }^{\mathrm{a}}$, Christina Dold ${ }^{\mathrm{a}}$, \\ Tsidi Agbeko Tamekloe ${ }^{\mathrm{f}}$, Christine S. Rollier ${ }^{\mathrm{a}}$, Hilary Watt ${ }^{\mathrm{h}}$, Abiba Banla Kere ${ }^{\mathrm{g}}$, Lisbeth M. Næss ${ }^{\mathrm{b}}$, \\ Andrew J. Pollard ${ }^{\mathrm{a}}$
}

\author{
a Oxford Vaccine Group, Department of Paediatrics, University of Oxford, and the NIHR Oxford Biomedical Research Centre, Oxford, UK \\ ${ }^{\mathrm{b}}$ Norwegian Institute of Public Health, Oslo, Norway \\ ${ }^{\mathrm{c}}$ Agence de Médecine Préventive, Paris, France \\ ${ }^{\mathrm{d}}$ Ecole des Hautes Etudes en Santé Publique, Rennes/Paris, France and Institut Pasteur, Paris, France \\ e Vaccine Evaluation Unit, Public Health England, Manchester, UK \\ ${ }^{\mathrm{f}}$ Division of Epidemiology, Ministry for Health, Togo \\ $\mathrm{g}$ Institute National d'Hygiene, Lome, Togo \\ ${ }^{\mathrm{h}}$ Department of Primary Care and Public Health, Imperial College, London, UK
}

\section{A R T I C L E I N F O}

Article history:

Received 5 November 2016

Received in revised form 10 January 2018

Accepted 11 January 2018

Available online 2 February 2018

\section{Keywords:}

Neisseria meningitidis

Meningococcal

Capsular group X

Polysaccharide

Bactericidal

Antibodies

\section{A B S T R A C T}

Background: Capsular group X N. meningitidis (MenX) has emerged as a cause of localized disease outbreaks in sub-Saharan Africa, but the human immune response following exposure to MenX antigens is poorly described. We therefore assessed the natural immunity against MenX in individuals who were living in an area affected by a MenX outbreak during 2007 in Togo, West Africa. During 2009, 300 healthy individuals (100 aged 3-5 years, 100 aged $13-19$ years and 100 aged $20-25$ years) were included in the study, and serum responses were compared with sera from age-matched controls from the U.K. and Burkina Faso.

Methods: MenX serum bactericidal antibody (SBA) was measured using rabbit complement, and antibodies against MenX polysaccharide (XPS) and outer membrane vesicles (XOMVs) were quantified by ELISA. Results: The proportion of Togolese individuals with an SBA titer of $\geq 8$ against the MenX strain was $29 \%$ (95\% confidence interval (CI) 18-41) among those aged 3-5 years, 34\% (95\% CI 9-60) among those aged $13-19$ years and 32\% (95\% CI 24-40) among those aged 20-25 years. These were significantly higher than observed in the control populations from the U.K (range 13-16\%) and Burkina Faso (range 2-6\%).

Conclusion: In Togolese individuals, the concentration of serum IgG against XPS was higher among the two older age groups as compared to the youngest age group. Antibody concentrations against MenX PS correlated significantly with SBA titers. This supports further development of a MenX PS based conjugate vaccine. Further studies are needed to verify the ability of MenX PS to induce SBA in humans.

(C) 2018 The Authors. Published by Elsevier Ltd. This is an open access article under the CC BY license (http:// creativecommons.org/licenses/by/4.0/).

\section{Introduction}

Meningitis epidemics in Africa have mainly been caused by Neisseria meningitidis of capsular group A, and most of the cases have occurred in a geographical area termed the Meningitis Belt; a region that stretches from Senegal in the West to Ethiopia in the East $[1,2]$. Each year the incidence of meningococcal disease

\footnotetext{
* Corresponding author at: Coalition for Epidemic Preparedness Innovation, Oslo, Norway.

E-mail address: gunnstein.norheim@fhi.no (G. Norheim).
}

peaks during the dry season, typically week 13 in the year, and in addition there are inter-annual epidemics that may include several neighbouring countries and involve up to 250,000 cases [2,3]. Capsular group X N. meningitidis (MenX) isolates were first documented in the meningitis belt in 1970 , but have recently emerged as a cause of more substantial localized disease outbreaks in subSaharan Africa in the last ten years, in both West and East Africa [4,5]. MenX disease was not raised as a significant concern until an outbreak in Niger in 2006 with over 800 cases and recognition of an increased incidence of MenX disease in Niger, Uganda, Kenya and Togo during 2006-08 [6]. In 2011, the World Health 
Organization (WHO) therefore decided to include MenX in the list of meningococci that are able to cause epidemics [7]. Of particular note is that MenX disease has shown a similar attack rate to capsular group A meningococci [4,8]. Since then, sporadic cases of MenX disease have been reported from the meningitis belt, notably in the Ivory Coast, Burkina Faso, Mali, Niger [9,10] and Ethiopia [11], with carriage also observed in Chad [12].

Following the implementation of the safe and highly effective capsular group A monovalent conjugate vaccine in 2010 in the meningitis belt, the main target for research now will be to eliminate disease caused by the other capsular groups prevailing in the Meningitis Belt, namely capsular groups C, W and X. There is currently no polysaccharide based vaccine commercially available to prevent MenX disease. Three polysaccharide-protein-conjugate vaccine candidates [13-15] and three outer membrane proteinbased vaccine candidates [16-20,44] have been shown to induce serum bactericidal antibodies (SBA) against MenX isolates from the African meningitis belt in animal studies. When progressing any of these into clinical immunogenicity trials, the functional activity of antibodies following vaccination will be a key endpoint.

Several seroprevalence studies in the African meningitis belt have described the relation between age-specific incidence of disease and the seroprevalence of SBA for capsular group A and W meningococcal disease, as well as with antibodies against purified polysaccharide [21-23], with substantial differences between capsular groups in the prevalence of natural immunity. The development of natural immunity against MenX and serological correlates of protection against this group is poorly understood, but is critical for assessment of vaccines.

To describe natural immunity among a population exposed to MenX, and to identify characteristics of putatively protected individuals, we performed a seroepidemiological study among healthy children and young adults living in Kara town, Togo, West Africa in 2009. This is an area affected by a MenX outbreak 2.5 years earlier, during 2007 [8]. Based on molecular typing performed on CSFs, the causative organism of the outbreak was a MenX strain of ST-181 expressing PorA P1.5 [8].

Togo is a West-African country with approximately 6.8 million inhabitants, and only in the northern part of the country the epidemiology is typical of the African meningitis belt [2]. Specifically, we compared seroprevalence data with the MenX age-specific meningitis incidence data from this area, to be able to interpret the data in light of possible cross-reactive immunity. We also compared these data with seroprevalence against MenX in serum samples from controls belonging to the same age group in Burkina Faso and the U.K.

\section{Methods}

\subsection{Seroprevalence study in Kara town, Togo}

A cross-sectional seroepidemiological study of the general population with one study visit per participant was performed in Kara during October 2009. Volunteers were recruited from the general population of Kara town, capital of Kozah district, the area experiencing the MenX outbreak during March 2007 [8]. Based on the presumption that seroprevalence varied by age, the study included healthy volunteers in three age strata: children aged 3-5 year (aged at least 1 year old during the outbreak season of 2007), teenagers (13-19 years of age) and young adults (20-25 years of age). In the absences of any pre-existing serological data on capsular group X, a sample size of 100 participants per age group was chosen for study feasibility. This samples size would provide prevalence estimates with 95\%-confidence intervals allowing a precision of \pm 5 to $\pm 9.8 \%$. Following a two-stage sampling design, we randomly selected neighbourhoods of Kara town and thereafter compounds. In each selected compound the individuals in the three target age groups were listed and one participant chosen randomly from each age group. Inclusion criteria were: healthy male or female individual aged 3-5 years old or 13-25 years old, residents of Kara since at least 2006, without severe disease, and who give informed consent. Exclusion criteria were: known bleeding disorder, receipt of treatment with immunosuppressive drugs and severe malnutrition. A venous blood sample $(10-20 \mathrm{~mL})$ was collected, processed and stored frozen in the national reference laboratory, Institut National d'Hygiène (INH) in the capital Lomé until shipment of aliquots to the U.K in November 2009. Data collected from participants were age, sex, location of residence, weight, height, meningococcal vaccination status, recent medical history and social contact pattern. The primary endpoint is the proportion of subjects with SBA titers of either greater or equal to $1: 8$ or $1: 128$.

\subsection{Controls from Burkina Faso and the United Kingdom}

Control sera were obtained from previous seroprevalence studies carried among healthy individuals aged 1-29 years in BoboDioulasso, Burkina Faso in 2003 [24] and 2006 [25], which used a similar protocol for population sampling and data collection. A subset of these, with similar age distribution as the Togolese samples, were randomly selected and served as African controls from an area with limited or no MenX disease. Sera were primarily from the 2006 study, and from the 2003 study if none age-matching sera were available from the 2006 study. Burkina Faso, which is bordering Togo to the north, is entirely within the meningitis belt and had never any MenX outbreak documented by the time of collection of the serum samples in this study. However, a serogroup A outbreak was ongoing during sample collection in 2006. Serum samples from children and teenagers, with a similar age distribution and living in the U.K., served as controls from countries with only sporadic or no reported MenX disease cases. These were randomly drawn from the collection of patient samples obtained by the Public Health England (PHE) Seroepidemiology Unit in or prior to 2009 [26]. The collection contains anonymised and stored residual sera derived from patients who provided a sample as part of medical care in England and Wales, and approximate the general population of England and Wales. Data available on each individual were age, sex, date of collection and source laboratory. Control subjects from U.K. and from Burkina Faso were selected by matching the available Togo study sera $(n=290)$ with the control serum banks by age strata. Following matching, serum aliquots were shipped to Oxford and Manchester for laboratory analysis.

\subsection{Quantitation of serum IgG against capsular group X antigens}

The assessment of IgG antibodies against XPS in human sera was performed as previously described [27]. The concentrations of IgG against MenX major outer membrane proteins in healthy human volunteer sera were assessed by use of ELISA, as previously described [28]. OMVs from MenX strain BF7/07 (ST-181, PorA P1.51,10-1 and FetA F1-31) were extracted by a deoxycholate based method, characterised by SDS-PAGE gel electrophoresis and electron microscopy as previously described [29]. Detergent extracted OMVs were chosen as coating antigen to enable quantification of the non-polysaccharide antibody responses, focussing on major outer membrane protein (PorA, FetA) antibodies and less on LPS and lipoproteins which are mainly removed during the detergent extraction process. 


\subsection{MenX SBA assay}

An SBA assay for capsular group X meningococcal bacteria was developed at the PHE's Vaccine Evaluation Unit in Manchester, U.K., with baby rabbit complement (Pel-Freeze, U.S.), as previously described [30], using a capsular group X sequence type (ST) 751 disease isolate from Burkina Faso in 1997 as the target strain (BF2/97) (PorA P1.5-1,10-1) [27]. This target strain from Burkina Faso was chosen due to its survival with complement sources (strain BF7/07 was not suitable; data not shown), and the lack of MenX outbreak strains from Togo suitable for SBA. The Togo outbreak strain (P1.5-1,10-1, no isolate available for sequence typing), the SBA target strain (BF2/97, from Burkina Faso 1997, ST-181 and PorA P1.5-1, 10-1, FetA F1-31) and the OMV strain (BF7/07, from Burkina Faso 2007, ST-751, PorA P1.5-1,10-1) all expressed the same PorA variant. These strains are likely different in other outer membrane proteins. A subset of $(n=10)$ human sera from Togolese individuals aged 13-19 years old and with rabbit complement SBA of $\geq 1: 128$ titers were also tested in an SBA assay against MenX isolate BF2/97 at the NIPH, Oslo, Norway, using a human complement source ("060") from a single human donor in Norway with no intrinsic bactericidal activity.

\subsection{Bacterial meningitis surveillance data in Togo}

Data on suspected meningitis surveillance in Kozah district, 2007-2008, were available from a hospital-based surveillance system set up by the Togolese Ministry of Health and the Agence de Médecine Préventive (AMP) [31], using PCR for confirmation of etiology. To estimate age-specific serogroup X meningitis incidence rates were estimated using demographic data from the 2010 Togo national census 2010

\subsection{Ethical approval}

Approvals for the study were obtained from the Oxford Tropical Research Ethics Committee (OXTREC reference number 40-09) on 24.07.2009, and from the "Comité de bioéthique pour la recherche en santé" (CBRS), Ministry of Health, Togo on 28.08.2009 (CBRS reference number reference number 1873/2009/MS/CAB/DGS/OPLET/ CBRS). National Research Ethics Service (NRES) approval for the sero-epidemiological surveillance of the National Immunisation programme of England and Wales (Research Ethics Committee number 05/Q0505/45) was granted by the Joint University College London/University College London Hospital (UCL/UCLH) Committees on the Ethics of Human Research.

\subsection{Statistical analysis}

The primary endpoint was the proportion of Togolese participants in the three age groups demonstrating MenX SBA geometric mean titers (GMT) either $\geq 8$ or $\geq 128$. Secondary endpoints were geometric mean concentrations (GMC) of IgG antibodies against XPS or XOMV in sera from the three age groups from Togo, Burkina Faso and the U.K. We defined malnutrition as weight for height below the 3rd percentile among < 6 -year-old children, and as body mass index below the 3rd percentile among individuals aged 13 years or older, using WHO growth standards [32]. Logistic and linear regression methods were used to compare seroprevalence and GMT between age groups, respectively. Correlation between SBA titers and $\operatorname{IgG}$ concentrations were analysed using Pearson correlation test on log-transformed data. The association between seroprevalence (SBA titer $\geq 128$ and anti-XPS $\operatorname{IgG} \geq 500 \mathrm{AU} / \mathrm{mL}$ ) and potential risk factors was evaluated by univariate and multivariate logistic regression models, with stepwise inclusion of variables with a P-value $<.2$ in the univariate model.

\section{Results}

3.1. MenX SBA and antigen-specific antibody seroprevalences across age groups in Togo

Among Togolese individuals, MenX SBA antibody levels were similar across the three age groups 3-5 years, 13-19 years and 20-15 years of age, with the GMT of SBA ranging between 6.0 and 6.7 and the prevalence of titers $\geq 128$ ranging between $21 \%$ and 26\% (Supplemental Table 1). The anti-XPS IgG concentrations were however significantly lower among children 3-5 years of age $(74.2 \mathrm{AU} / \mathrm{mL})$ compared with individuals aged $13-19$ years $(234.1 \mathrm{AU} / \mathrm{mL}$ ) or $20-25$ years $(293.9 \mathrm{AU} / \mathrm{mL}$ ) (both $p<.001$ ), (Supplemental Table 2). Likewise, the anti-XOMV IgG concentrations (Supplemental Table 3) were lower among children 3-5 years of age $(67,124 \mathrm{AU} / \mathrm{mL})$ compared with individuals aged $13-19$ years $(123,327 \mathrm{AU} / \mathrm{mL})$ or $20-25$ years $(113,978 \mathrm{AU} / \mathrm{mL})(p<.001$ for the latter). Risk factors reported among Togolese individuals are reported in Supplemental Table 5. Of these, age group was significantly associated with XPS IgG concentration $\geq 500 \mathrm{AU} / \mathrm{mL}$ (odds ratio (OR) versus age group 3-5 yrs was 6.72 (95\% CI 2.44-18.54) for age group 13-19 years; and 7.84 (95\% CI 2.90-21.17 for age group 20-25 years)) but not with SBA titer $\geq 128$.

\subsection{MenX SBA GMT and seroprevalence country comparison}

Comparing GMT of MenX SBA among Togolese participants aged 3-5 years with that in control participants, this was higher among the Togolese participants (6.4, range 2.9-14.1) than among those from the U.K. (1.9, range 1.4-2.5) and Burkina Faso (1.1, range 1.0-1.2) (Supplemental Table 1). The same mean titer pattern was observed for the other age groups as well. Applying various thresholds for MenX SBA revealed differences in country comparisons, and with the most stringent threshold $(\geq 128)$ we found higher proportions of Togolese participants demonstrating MenX seroprevalence (26\%) than for those from the U.K. (5\%) and Burkina Faso (0\%). There were no differences in the SBA GMT between the three age groups within each of the three countries (Supplemental Table 1).

\subsection{MenX antigen specific IgG seroprevalence country comparison}

Country comparison of anti-XPS IgG yielded a similar pattern as for rSBA, depending on the threshold applied for the comparison (Supplemental Table 2). In the age groups 13-19 and 20-25 years, the GMC among Togolese individuals was nearly double the GMC among U.K. individuals. On the contrary, among individuals aged 3-5 years, the GMC of Burkina Faso participants was nearly twice that observed among U.K. and Togolese participants. The lower threshold (anti-XPS IgG $\geq 100 \mathrm{AU} / \mathrm{mL}$ ) indicated a high proportion of participants exhibiting a presence of anti-XPS IgG in all countries and age groups (from 35 to $90 \%$ ), possibly caused by crossreacting organisms. Applying a higher threshold (XPS IgG $\geq 500 \mathrm{~A}$ $\mathrm{U} / \mathrm{mL}$ ) (Supplemental Table 2) indicated that natural exposure to MenX may induce higher IgG concentration in $30 \%$ of the adult population (20-25 years of age in 2009; 18-23 years in 2007).

The levels of anti-OMV IgG were significantly lower among Togolese children aged 3-5 years (GMC 67,124 AU/mL) compared to controls from Burkina Faso from the same age group (GMC 107,040) (Supplemental Table 3). The same pattern was found for the proportion of participants with a level of anti-XOMV IgG $\geq 10,000 \mathrm{AU} / \mathrm{mL} ; 24 \%$ among Togolese versus $48 \%$ among controls (Supplemental Table 3). Among adolescents and adults, no differences were found between individuals from the two countries in 


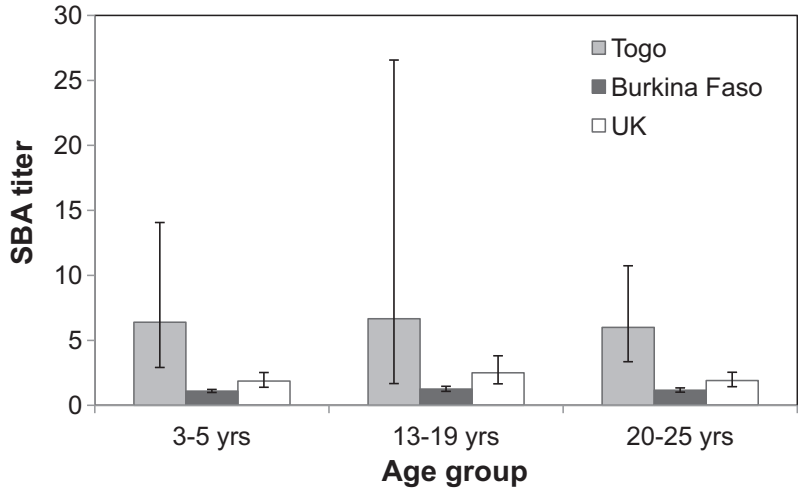

Fig. 1. Immunity against MenX in sera collected from Togolese participants in 2009, two years after a localized MenX outbreak in the same area. Geometric mean concentrations of SBA titers across age groups. Bars indicate 95\% confidence intervals.

GMC ( $\sim 100,000 \mathrm{AU} / \mathrm{mL})$ and proportion with anti-OMV $\operatorname{IgG} \geq 10$, $000 \mathrm{AU} / \mathrm{mL}(\sim 50 \%)$.

\subsection{Relationship between MenX SBA seroprevalence and disease incidence in Togo}

The seroprevalence of putatively protective MenX SBA titers (Fig. 1) and anti-XPS-IgG (Fig. 2) per age group in Kara in 2009 were compared graphically with historic incidence (cases per 10,000 persons) of MenX disease in the same age groups and area observed during 2007 (Figs. 3 and 4). The highest disease incidence was seen in children aged 3-5 years of age, and this co-incided with a low level of antibodies against MenX PS and OMVs. The disease incidence was lowest among adults aged 26-29 years, and this group had the highest proportion of anti-XPS IgG. There hence appeared to be an inverse relation between age-specific incidence of disease and seroprevalence of anti-XPS IgG.

\subsection{Correlation between SBA and IgG results}

The specificity of the antibodies responsible for causing the observed SBA could to some degree be explained by IgG against MenX polysaccharide, as a significant and strong $(r=0.63)$

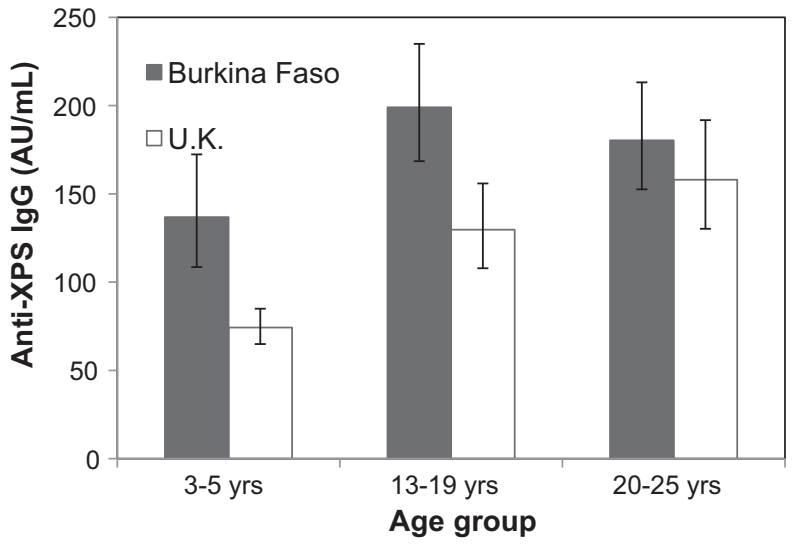

Fig. 2. Total IgG against purified serogroup X N. meningitidis polysaccharide in sera from Togolese, Burkina Faso and U.K. participants (geometric mean concentration and $95 \%$ confidence intervals).

association was detected among sera from Togolese aged 13-19 years (Supplemental Table 4, Fig. 5). Moderate correlations between SBA and XPS IgG were observed among Togolese aged 20-25 years of age and Burkina Faso participants aged 13-19 years of age. Significant correlations ( $\mathrm{p}<.001$ ) between SBA and XPS IgG were also observed for the older age groups $13-19$ years $(r=0.521)$ and 20-25 years $(r=0.416)$ when analysed across countries. Similarly, significant correlations between individual SBA titers and anti-OMV IgG concentrations were observed for Togolese individuals aged $13-19$ and $20-25$ years $(r=\sim 0.4)$.

\section{Discussion}

\subsection{Proportion of participants with MenX SBA highest in adolescents} and adults

In this study evaluating the seroprevalence of antibodies against MenX in a Togolese community that has experienced an MenX meningitis outbreak 2.5 years prior to sampling, we found a low seroprevalence of SBA against MenX, and that the SBA seroprevalence was significantly lower among children aged 3-5 years than among and adolescents or adults. A high seroprevalence of 70

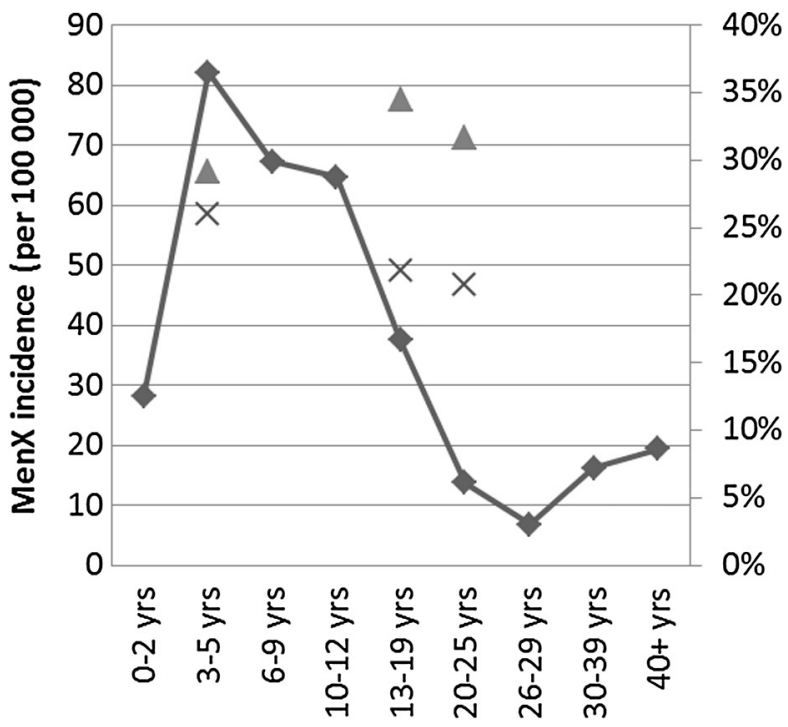

Fig. 3. Age-related SBA seroprevalence against capsular group $X$ in Togo in 2009, versus incidence of MenX disease per of the 3 age groups in Togo 2007. 

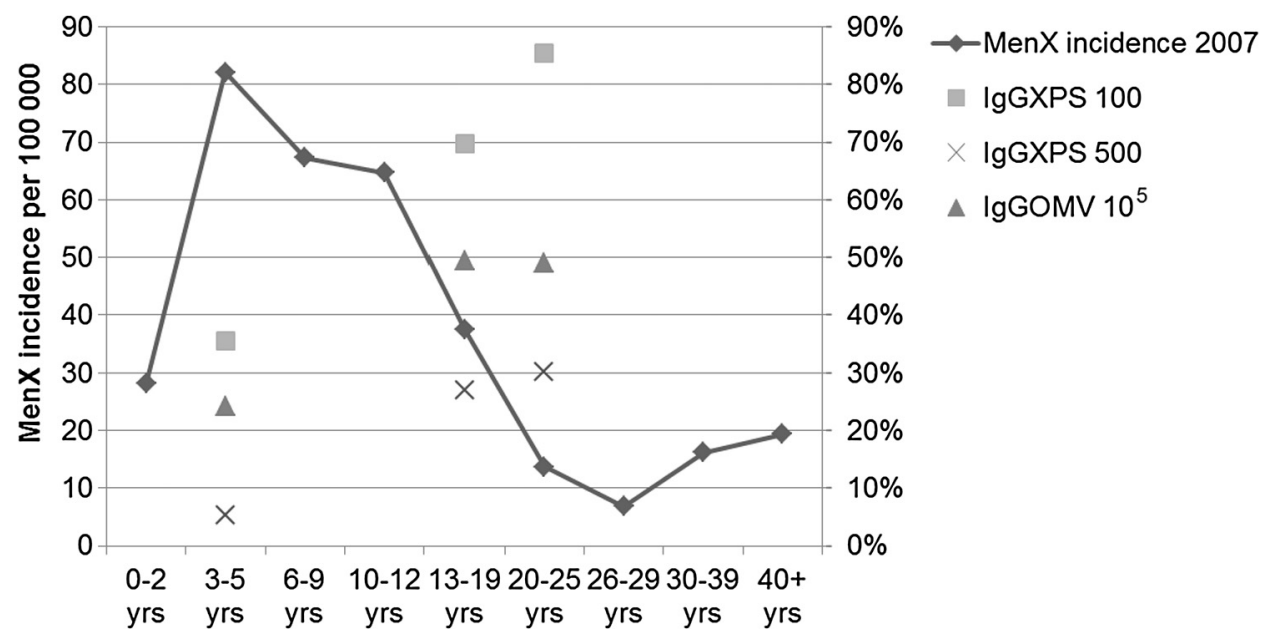

Fig. 4. Age-related serum IgG thresholds against XPS or OMVs in Togo versus incidence of MenX disease per each age group in Togo in March 2007.

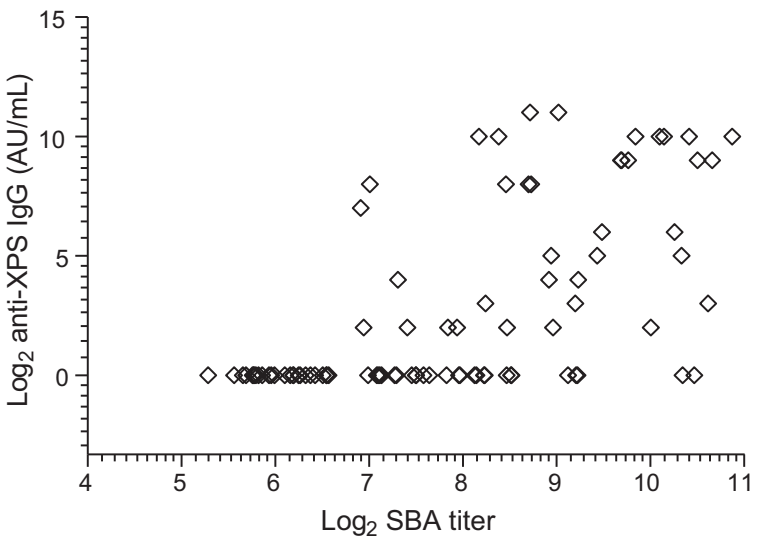

Fig. 5. Correlation between MenX SBA and IgG against MenX polysaccharide concentrations (both $\log _{2}$-transformed) in sera from Togolese participants aged 1319 years of age $(r=0.6288, n=90)$.

and $85 \%$ was however found for anti-XPS $\operatorname{IgG} \geq 100 \mathrm{AU} / \mathrm{mL}$ among adolescents and adults, respectively. The Togolese children in this study were sampled at 3-5 years of age (October 2009), but were aged $0-2$ years at the time of the serogroup $\mathrm{X}$ outbreak (March 2007). Despite the young age, the Togolese children still had a higher percentage of subjects with SBA titers $>1: 8$ than subjects of the same age group in the control populations (Supplemental Table 1). Pronounced differences in seroresponses between sera from the three countries were only evident when applying higher thresholds (either SBA $\geq 128$ or anti-XPS $\operatorname{IgG} \geq 500 \mathrm{AU} / \mathrm{mL}$ ). The Togolese population showed substantially higher prevalences than the two control populations from Burkina Faso and the U.K, in particular adolescents and adults.

The seroprevalences against meningococcal capsular polysaccharides are widely diverging, as e.g. in Saudi Arabia and Burkina Faso where naturally occurring concentrations of antibodies against capsular groups $\mathrm{C}, \mathrm{Y}$ and $\mathrm{W}$ are much lower than those observed against capsular group A [24,35]. Putatively protective rabbit-complement SBA titers are commonly observed for approximately $20 \%$ of African individuals $>2$ years of age against capsular group W [23], and an age-dependent increase in immunity is seen for capsular groups $C$ and $Y$ at least up to 4 years of age [24,35]. Pre-existing anti-capsular group A polysaccharide antibody concentrations are usually higher in Africans than in individuals from Europe and North America [36], posing a challenge for interpretation of relative immunogenicity [37].
4.2. Specificity of bactericidal antibodies and origin of MenX specific immunity

The in vitro SBA activity is mediated by antibodies directed against multiple meningococcal antigens, in particular the polysaccharide capsule, but may also be directed against outer membrane proteins and lipooligosaccharide. Given the lack of a strong correlation between XPS IgG and SBA in the study population as a whole (with the exception of those aged 13-19 years), and uncertainties on MenX PS assay specificity, inferences on causality between these antibodies and disease susceptibility or protection is however uncertain. Correlation analyses to elucidate the specificity of the observed rSBA titers showed that anti-XPS IgG could explain a significant proportion of the SBA among sera from Togolese aged 13-19 years and 20-25 years, as well as among Burkina Faso participants aged 13-19 years (Supplemental Table 4), supporting vaccine development based on capsular group $\mathrm{X}$ polysaccharides, with conjugate vaccines being the most promising option $[13,14]$. The apparent age-related increase in proportions of Togolese individuals with a high antibody titer against MenX PS or MenX OMV did not correlate with the proportion with an SBA titer $\geq 128$; which was relatively constant across age groups (21-26\%). This could be explained by the choice of baby rabbit serum as a complement source in the SBA assay; known to result in higher SBA titers and non-specific activity as compared with human complement. Future studies should however assess responses in human convalescent sera from MenX patients and address the impact of the SBA complement source. A preliminary exploration of the effect of the complement source on SBA indicated no covariation between SBA titers obtained with human and rabbit complement (Supplemental Table 6). Our study found that there was background immunity against MenX antigens (XPS or XOMVs) in both control populations (Burkina Faso and U.K.). This may be explained by the induction of cross-reactive antibodies by non-MenX organisms, as previously described for other meningococci [38-40]. In the U.K., the presence of 5-9\% of responders with an SBA titer of $\geq 128$ may be due to non-specific immunity from exposure to non-MenX organisms, perhaps capsular groups $C$ or B among the 20-25 yrs participants in U.K. The sera from UK were collected in 2009 , and the individual exposure to meningococcal carriage prior to 2009 for these individuals is not known. However, isolated MenX cases have been reported in the U.K. in the period 19992006 [33] and carriage studies among adolescents from U.K. in the period 1999-2001 showed 0.21\% MenX carriage (102 isolates among 47765 participants, $\sim 1.1 \%$ of all meningococcal isolates) 
[34]. The assays chosen have limited ability to unambiguously dissect the basis of antigen-specific immunity in Togo, and this prevents a clear determination of anti-OMV bactericidal activityThe anticapsular specificity of the assay and potential cross-reactivity between MenX and MenA PS should be further explored, as we cannot exclude a potential cross-reaction between MenX and MenA PS.

\subsection{Comparing serological status across volunteers from Togo, Burkina} Faso and the U.K

A similar pattern was observed in all three countries when applying high thresholds for rSBA (titer $\geq 128$ ) and anti-XPS IgG (concentration $\geq 500 \mathrm{AU} / \mathrm{mL}$ ), with a higher proportion of adolescents or adults with immunity than children. However, for XOMV-IgG (using a threshold of $\geq 10,000 \mathrm{AU} / \mathrm{mL}$ ) the lower proportion with high levels of anti-XOMV-IgG in Togolese compared with Burkina Faso children was unexpected. This may indicate low MenX carriage among Togolese children in 2007 and possibly long lasting cross-reactive antibody responses to outer membrane antigens among Burkina Faso participants; where outbreaks have been more frequent and widespread than in Togo.

The observation that there was a lack of MenX seroprevalence in the youngest age group in 2009, 2 years after the 2007 outbreak (Figs. 3 and 4), could either be a result of a lack of exposure in this age group, a lack of immune response or a short duration of immunity against MenX. For the older groups, there was an apparent persistence of MenX seroprevalence 2 years after the 2007 outbreak. There were a high proportion of Burkina Faso participants (from 2006) who had SBA titers $\geq 4$ against MenX which may be explained by non-specific immunity from MenA which showed a high prevalence in Burkina in 2006 [25].

These data suggest that exposure to MenX, presumably through nasopharyngeal colonisation, has occurred in Togo. This presumably indicates transmission in the population, but could also be due to cross-reactive antibodies. Despite the increase in overall antibody concentration with age, the similarity in SBA titer in all age strata suggests that this exposure may not guarantee the induction of functional antibodies, assuming that rabbit complement SBA is a reflection of protection against MenX disease in humans. This may not be the case, as indicated in a recent study assessing human antibody responses for a serogroup A conjugate vaccine [45], and future studies should assess SBA using both human and rabbit complement. As a new area of serologic evaluation, the study of immunity to serogroup $X$ would be improved with the inclusion of hSBA data. The lack of correlation between MenX hSBA and rSBA titers observed in our limited subset of sera assessed (Supplemental Table 6) parallels findings for e.g. serogroup A [45]. The IgG data for Burkina Faso and UK, where serogroup $\mathrm{X}$ has been rare suggest that IgG alone is unlikely to predict serogroup specific protective immunity.

The current study demonstrated that MenX PS is likely immunogenic in humans and can induce bactericidal antibodies. Further studies in individuals with individually verified exposure to MenX antigens are needed, i.e. among individuals asymptomatic MenX carriers, and patients surviving MenX disease. Since MenX disease has so far only been responsible for sporadic disease in Western countries and limited outbreaks in Africa, there has been no commercial interest in vaccine development. Although predicting future disease outbreaks is not possible, the advent of a MenX strain of ST-7 causing an invasive disease case in China in 2013 [41] may serve as a warning signal - strains from the ST-5 clonal complex are among the most succesful strains in causing pandemic waves of meningococcal disease [42]. Development of a capsular group $X$ vaccine [27] is hence urgently needed to control or prevent potential future outbreaks. A multivalent capsular group ACYWX conjugate vaccine (i.e. tetanus toxoid for the Men A and X polysaccharides and CRM197 for the Men C, Y and W polysaccharides) is currently in development by the Serum Institute of India and the Project for Appropriate Technology in Health (PATH), and was recently reported to elicit high rSBA titers against MenX in a phase I trial among U.S. adults [43]. This is an incredibly promising development for meningococcal disease prevention in the African Meningitis Belt.

\section{Authors' contributions}

All authors participated in the design or implementation or analysis, or interpretation of the study; and the development of this manuscript. The work described was carried out in accordance to ICMJE recommendations for conduct, reporting, editing and publication of scholarly work in medical journals. The corresponding author had final responsibility to submit for publication.

GN, AJP, JEM and RB designed the study; JEM, BL, ID, TAT, ABK performed the field study in Togo and collected and assembled demographic data; GN, HF, RB, OX, JN, RR, CD, CR and LMN carried out the laboratory work, JEM, GN, HW, AJP, CR, HF, RB, OX, LMN analysed study data; GN, JEM and AJP interpreted study data, GN wrote the first draft, all authors edited. All the authors had full access to the data and gave final approval before submission.

\section{Funding}

This work was funded by a research grant from the Meningitis Research Foundation, U.K. (Grant number 0906.1) and supported by the Oxford Partnership Comprehensive Biomedical Research Centre with funding from the NIHR Biomedical Research Centre Programme.

\section{Role of the funding source}

The funders had no role in the design of the study; collection, analysis and interpretation of data; writing of the report; or the decision to submit the paper for publication. The corresponding author had full access to all the data from the study and had final responsibility for the decision to submit for publication.

\section{Conflict of interest}

AJP has previously acted as chief and principal investigator for clinical trials conducted on behalf of Oxford University, sponsored by vaccine manufacturers (Novartis Vaccines, GlaxoSmithKline, Sanofi-Pasteur, Sanofi-Pasteur MSD, and Pfizer Vaccines), but does not receive any personal payment from them. RB and HF perform contract research on behalf of Public Health England for GSK, Novartis, Pfizer, Sanofi Pasteur and the Serum Institute of India. B.-M. N. L. and I.D. is employed by AMP, which receives grant support from Crucell, GlaxoSmithKline (GSK), Merck, Novartis, Pfizer, and Sanofi Pasteur. J.M. was previously employed by AMP.

\section{Acknowledgements}

AJP is a Jenner Institute Investigator and a James Martin senior fellow. Prof. D. A. Caugant, WHO Collaborating Centre for Reference and Research on Meningococci, Norwegian Institute of Public Health, Oslo, Norway, is thanked for the capsular group X isolates from Burkina Faso and providing sequence and serotype data on these. CR is a Jenner Institute Investigator and Oxford Martin fellow. Mrs Kalpana Sinnadurai is thanked for the human complement based serum bactericidal assay testing at NIPH. 


\section{Appendix A. Supplementary material}

Supplementary data associated with this article can be found, in the online version, at https://doi.org/10.1016/j.vaccine.2018.01. 031.

\section{References}

[1] Lapeyssonnie L. Cerebrospinal meningitis in Africa. Bull World Health Organ 1963;28(Suppl):1-114.

[2] Molesworth AM, Thomson MC, Connor SJ, Cresswell MP, Morse AP, Shears P, et al. Where is the meningitis belt? Defining an area at risk of epidemic meningitis in Africa. Trans R Soc Trop Med Hyg 2002;3:242-9.

[3] Greenwood B. Manson Lecture. Meningococcal meningitis in Africa. Trans R Soc TropMed Hyg 1999;4:341-53.

[4] Boisier P, Nicolas P, Djibo S, Taha MK, Jeanne I, Mainassara HB, et al. Meningococcal meningitis: unprecedented incidence of serogroup X-related cases in 2006 in Niger. Clin Infect Dis 2007:5:657-63.

[5] Materu S, Cox HS, Isaakidis P, Baruani B, Ogaro T, Caugant DA. Serogroup X meningococcal disease, Western Kenya. Emerg Infect Dis 2007;16:944-5.

[6] Xie O, Pollard A, Mueller JE, Norheim G. Emergence of serogroup X meningococcal disease in Africa: need for a vaccine. Vaccine 2013;27:2852-61.

[7] World Health Organization. Fact sheet $\mathrm{N}^{\circ} 141$. Meningococcal meningitis. Geneva, Switzerland; 2011. http://www.who.int/mediacentre/factsheets/ fs141/en/.

[8] Delrieu I, Yaro S, Tamekloe TA, Njanpop-Lafourcade BM, Tall H, Jaillard P, et al Emergence of Epidemic Neisseria meningitidis Serogroup X Meningitis in Togo and Burkina Faso. PLoS One 2011;5:e19513.

[9] World Health Organization. Meningitis Weekly Bulletin, Week 25 Meningococcal disease control in countries of the African meningitis belt 2014. West-Africa Inter-Country Support Team. Ouagadougou, Burkina Faso: World Health Organization; 2015.

[11] Mihret W, Lema T, Merid Y, Kassu A, Abebe W, Moges B, et al. Surveillance of Bacterial Meningitis, Ethiopia, 2012-2013. Emerg Infect Dis 2016;22(1):75-8.

[12] MenAfriCar consortium. The diversity of Meningococcal Carriage across the African Meningitis belt and the impact of vaccination with a group A Meningococcal conjugate vaccine. J Infect Dis 2015;8:1298-307.

[13] Micoli F, Romano MR, Tontini M, Cappelletti E, Gavini M, Proietti D, et al Development of a glycoconjugate vaccine to prevent meningitis in Africa caused by meningococcal serogroup X. Proc Natl Acad Sci U S A 2013;47:19077-82.

[14] Chilukuri SR, Reddy P, Avalaskar N, Mallya A, Pisal S, Dhere RM. Process development and immunogenicity studies on a serogroup X meningococcal polysaccharide conjugate vaccine. Biologicals 2014;3:160-8.

[15] Morelli L, Cancogni D, Tontini M, Nilo A, Filippini S, Costantino P, et al. Synthesis and immunological evaluation of protein conjugates of Neisseria meningitidis $\mathrm{X}$ capsular polysaccharide fragments. J Org Chem 2014;10:2367-76.

[16] Beernink PT, Caugant DA, Welsch JA, Koeberling O, Granoff DM. Meningococcal factor $\mathrm{H}$-binding protein variants expressed by epidemic capsular group A, W135 , and X strains from Africa. J Infect Dis 2009;9:1360-8.

[17] Norheim G, Tunheim G, Naess LM, Bolstad K, Fjeldheim ÅK, Garcia L, Cardoso D, Caridad Z, Acevedo R, Rosenqvist E. 2012. A trivalent outer membrane vesicle (OMV) vaccine against serogroup $A, W-135$ and $X$ meningococcal disease. In: Proceedings from XVIIIth International Pathogenic Neisseria Conference, September 2012. Würzburg, Germany.

[18] Pajon R, Fergus AM, Koeberling O, Caugant DA, Granoff DM. Meningococcal factor $\mathrm{H}$ binding proteins in epidemic strains from Africa: implications for vaccine development. PLoS Negl Trop Dis 2011;9:e1302.

[19] Pinto VB, Burden R, Wagner A, Moran EE, Lee CH. The development of an experimental multiple serogroups vaccine for Neisseria meningitidis. PLoS One 2013;11:e79304.

[20] Tunheim G, Naess LM, Acevedo R, Fjeldheim AK, Bolstad K, Garcia L, et al. Preclinical immunogenicity study of trivalent meningococcal AWX-OMV vaccines for the African meningitis belt. Vaccine 2014;49:6631-8.

[21] Manigart O, Trotter C, Findlow H, Assefa A, Mihret W, Moti Demisse T, et al. A seroepidemiological study of serogroup A Meningococcal infection in the African Meningitis belt. PLoS One 2016;2:e0147928.

[22] Trotter CL, Yaro S, Njanpop-Lafourcade BM, Drabo A, Kroman SS, Idohou RS, et al. Seroprevalence of bactericidal, specific $\operatorname{Ig} G$ antibodies and incidence of meningitis due to group A Neisseria meningitidis by age in Burkina Faso 2008. PLoS One 2013:2:e55486.

[23] Mueller JE, Borrow R, Gessner BD. Meningococcal serogroup W135 in the African meningitis belt: epidemiology, immunity and vaccines. Expert Rev Vaccines 2006;3:319-36.

[24] Mueller JE, Yaro S, Traore Y, Sangare L, Tarnagda Z, Njanpop-Lafourcade BM, et al. Neisseria meningitidis serogroups A and W-135: carriage and immunity in Burkina Faso, 2003. J Infect Dis 2006;6:812-20.

[25] Mueller JE, Yaro S, Njanpop-Lafourcade BM, Drabo A, Idohou RS, Kroman SS, et al. Study of a localized meningococcal meningitis epidemic in Burkina Faso: incidence, carriage, and immunity. J Infect Dis 2011;11:1787-95.

[26] Trotter CL, Findlow H, Borrow R. Seroprevalence of serum bactericidal antibodies against group W135 and Y meningococci in England in 2009. Clin Vaccine Immunol 2012;2:219-22.

[27] Xie O, Bolgiano B, Gao F, Lockyer K, Swann C, Jones C, et al. Characterization of size, structure and purity of serogroup X Neisseria meningitidis polysaccharide, and development of an assay for quantification of human antibodies. Vaccine 2012;40:5812-23.

[28] Norheim G, Aseffa A, Yassin MA, Mengistu G, Kassu A, Fikremariam D, et al Serum antibody responses in Ethiopian meningitis patients infected with Neisseria meningitidis serogroup A sequence type 7. Clin Vaccine Immunol 2007;4:451-63.

[29] Norheim G, Aase A, Caugant DA, Hoiby EA, Fritzsønn E, Tangen T, et al Development and characterisation of outer membrane vesicle vaccines against serogroup A Neisseria meningitidis. Vaccine 2005;29:3762-74.

[30] Maslanka SE, Gheesling LL, Libutti DE, Donaldson KB, Harakeh HS, Dykes JK, et al. Standardization and a multilaboratory comparison of Neisseria meningitidis serogroup $\mathrm{A}$ and $\mathrm{C}$ serum bactericidal assays. The multilaboratory study group. Clin Diagn Lab Immunol 1997;2:156-67.

[31] Adjogble KL, Lourd M, Njanpop-Lafourcade BM, Traore Y, Hlomaschi AF, Amegatse KA, et al. The epidemiology of Neisseria meningitidis meningitis in Togo during 2003-2005. Vaccine 2007;25(Suppl):A47-52.

[32] World Health Organization. WHO Child Growth Standards based on length/ height, weight and age. Acta Paediatr 2006;450:76-85.

[33] Network EU-IBIS. Invasive Neisseria meningitidis in Europe 2006. London: Health Protection Agency; 2006.

[34] Ibarz-Pavon AB, MacLennan J, Andrews NJ, Gray SJ, Urwin R, Clarke SC, et al. Changes in serogroup and genotype prevalence among carried meningococci in the United Kingdom during vaccine implementation. J Infect Dis 2011;7:1046-53.

[35] Al-Mazrou Y, Khalil M, Borrow R, Balmer P, Bramwell J, Lal G, et al. Serologic responses to ACYW135 polysaccharide meningococcal vaccine in Saudi children under 5 years of age. Infect Immun 2005;5:2932-9.

[36] Amir J, Louie L, Granoff DM. Naturally-acquired immunity to Neisseria meningitidis group A. Vaccine 2005;8:977-83.

[37] Idoko OT, Okolo SN, Plikaytis B, Akinsola A, Viviani S, Borrow R, et al. The impact of pre-existing antibody on subsequent immune responses to meningococcal A-containing vaccines. Vaccine 2014;33:4220-7.

[38] Robbins JB, Schneerson R, Xie G, Hanson LA, Miller MA. Capsular polysaccharide vaccine for Group B Neisseria meningitidis, Escherichia coli K1, and Pasteurella haemolytica A2. Proc Natl Acad Sci U S A 2011:44:17871-5.

[39] Glode MP, Robbins JB, Liu TY, Gotschlich EC, Orskov I, Orskov F. Crossantigenicity and immunogenicity between capsular polysaccharides of group C Neisseria meningitidis and of Escherichia coli K92. J Infect Dis 1977;1:94-104.

[40] Vann WF, Liu TY, Robbins JB. Bacillus pumilus polysaccharide cross-reactive with meningococcal group A polysaccharide. Infect Immun 1976;6:1654-62.

[41] Pan J, Yao P, Zhang H, Sun X, He H, Xie S. The case of a new sequence type 7 serogroup X Neisseria meningitidis infection in China: may capsular switching change serogroup profile? Int J Infect Dis 2014;29:62-4.

[42] Nicolas P, Norheim G, Garnotel E, Djibo S, Caugant DA. Molecular epidemiology of Neisseria meningitidis isolated in the African Meningitis Belt between 1988 and 2003 shows dominance of sequence type 5 (ST-5) and ST-11 complexes. J Clin Microbiol 2005;10:5129-35.

[43] LaForce M. Progress on MenACWYX vaccine: phase 1 trial results. In: Conference Abstract. Meningitis Research Foundation: meningitis and septicaemia in children and adults. 14-15 November. London, U.K.; 2017.

[44] Hong E, Giuliani MM, Deghmane AE, Comanducci M, Brunelli B, Dull P, et al. Could the multicomponent meningococcal serogroup B vaccine (4CMenB) control Neisseria meningitidis capsular group X outbreaks in Africa? Vaccine 2013;31:1113-6.

[45] Price GA, Hollander AM, Plikaytis BD, Mocca BT, Carlone G, Findlow H, et al. Human complement bactericidal responses to a group a meningococcal conjugate vaccine in africans and comparison to responses measured by 2 other group a immunoassays. Clin Infect Dis 2015;61(Suppl 5):S554-62. 